\title{
Les incisives de bouquetin perforées de Praileaitz I (Deba, Gipuzkoa) Analyse archéozoologique et technologique
}

\author{
Praileaitz leko (Deba, Gipuzkoa) basahuntzen ebakortz zulatuak \\ Analisi arkeozoologikoa eta teknologikoa \\ The perforated ibex incisors from Praileaitz I (Deba, Gipuzkoa) \\ Archaeozoological and technological analysis

\section{Los dientes de cabra perforados de Praileaitz I (Deba, Gipuzkoa) Analísis arqueozoológico y tecnológico}

MOTS-CLÉ: Parure, Magdalénien, Microscopie, Décoration, Traces d'usure.

GAKO-HITZAK: Norbanakoen apaingarriak, Madeleine, Mikroskopia, Apaindura, Trazeologia.

KEYWORDS: Personal ornaments, Magdalenian, Microscopy, Decoration, Use-wear

PALABRAS CLAVE: Adornos personales, Magdaleniense, Microscopia, Decoración, Traceología.

Marian VANHAEREN(1), Francesco D'ERRICO(1-2)

\section{RÉSUMÉ}

Les trois incisives biperforées découvertes les unes près des autres à l'entrée de la Grotte de Praileaitz I proviennent de deux jeunes bouquetins et faisaient vraisemblablement partie à l'origine d'un même objet de parure, qui devait comporter d'autres ornements. Ces résultats étayent l'hypothèse d'une occupation brève du site pendant le Magdalénien inférieur cantabrique.

\section{LABURPENA}

Praileaitz I haitzuloaren sarreran, elkarrengandik hurbil, bi basahuntz gazteren hiru ebakortzak aurkitu ziren, eta ziur aski zulo bina zituztenak, eta ziur aski beste ale gehiago ere izango zituen apaingarri baten parte izango ziren. Emaitza hauek, Behe Madeleine kantauriarrean, gunearen okupazio labur baten hipotesia baieztatzen dute.

\section{ABSTRACT}

We submit three perforated ibex incisors discovered close to one another in a Lower Magdalenian level at the entrance of the Praleaitz I Cave to an archaeozoological and technological analysis to determine the species and age of the animal, the anatomical position of the teeth, the manufacturing techniques, the objects' lifespan, and the possible reasons for their loss or disposal. Results indicate that the three incisors come from two different young adult ibexes. Microscopic observation allows for the identification of probable traces of extraction, markings made to position the tool used to perforate the teeth, and features demonstrating that the perforations were carried out by rotation and from two sides. We also identified decorative lines and notches, residue of red pigment, and use-wear on the perforation edges and on the apical region of the root. Comparison of these features with those observed on three perforated ibex incisors from Aitzbitarte, coming from a single animal, suggests that the incisors from Praileaitz I were originally integrated in a single large beadwork that included other ornaments, and of which the thread broke, probably at the site. These findings are in accordance with the hypothesis of a short occupation of the site during the Cantabrian Lower Magdalenian.

\section{RESUMEN}

Los tres incisivos con doble perforación descubiertos, cerca los unos de los otros, en la entrada de la cueva de Praileaitz I provienen de dos jóvenes cabras montesas y, probablemente, eran originalmente parte del mismo objeto de adorno, que debía incluir otros elementos de adorno. Estos resultados apoyan la hipótesis de una breve ocupación del sitio durante el Magdaleniense Inferior cantábrico.

\footnotetext{
(1) UMR 5199 De la Préhistoire à l'Actuel : Culture, Environnement, Anthropologie (PACEA), CNRS, Université de Bordeaux, Bâtiment B18, Allée Geoffroy St Hilaire, CS50023, F-33615 PESSAC CEDEX. marian.vanhaeren@u-bordeaux.fr (2) DST-NRF Centre of Excellence in Palaeosciences, Evolutionary Studies Institute, University of the Witwatersrand, Private Bag 3, WITS 2050, Johannesburg, South Africa.
} 


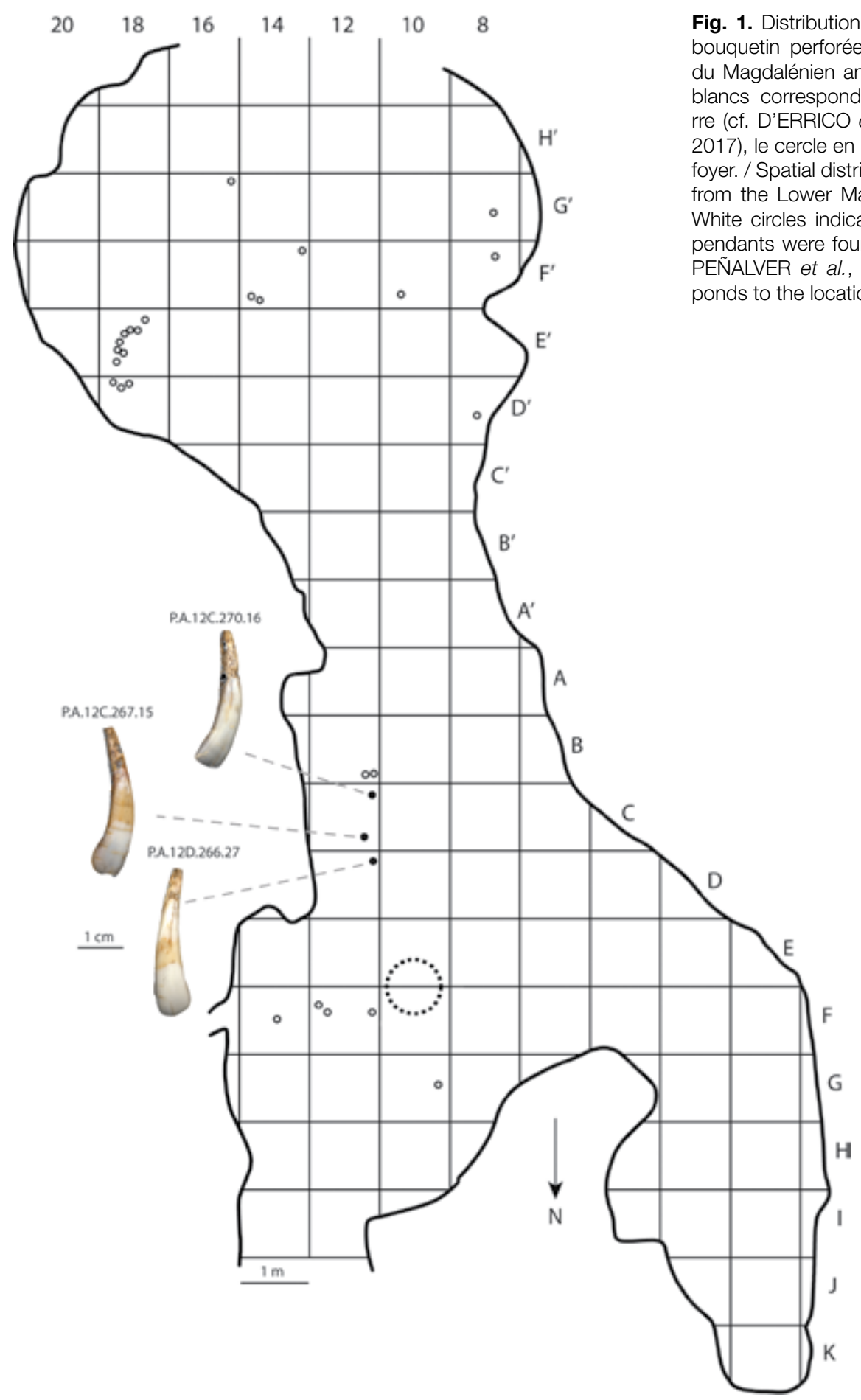

\section{1.- INTRODUCTION}

Les travaux menés au cours de la dernière décennie sur les objets de parure préhistoriques ont mis en évidence que le contexte archéologique et la matière dans laquelle ils ont été réalisés déterminent en grande partie leur potentiel d'information (VANHAEREN, 2002, ÁLVAREZ FERNANDEZ, 2006, BONNARDIN, 2009, RIGAUD,
2011). Les parures associées à des sépultures primaires, les lots d'ornements découverts groupés et les parures issues de sites occupés pendant une courte durée représentent des cas privilégiés. Ils garantissent en effet une certaine contemporanéité qui offre, à l'échelle des temps préhistoriques, une image instantanée des pratiques liées à la parure chez un seul groupe humain, voire un seul individu. Aussi, certaines matières premières se 
prêtent plus que d'autres à l'identification de comportements propres à une personne ou à un groupe restreint. Ainsi, les dents ou les valves de coquillages, qui possèdent des traits phénotypique distinctifs ou qui, par leur usure, patine, âge, facettes de contact, peuvent être reliées à un seul organisme, donnent la garantie d'une contemporanéité. Elles permettent également, par l'analyse des traces d'aménagement et d'usure, de saisir la variabilité individuelle ou d'un groupe restreint dans la fabrication et les utilisations des objets. Cette approche, appliquée à des parures associées à des ensembles clos datés du Paléolithique et du Mésolithique (VANHAEREN, D'ERRICO, 2003; RIGAUD, 2011; HENSHILWOOD, D'ERRICO, 2005), a permis de proposer des hypothèses sur la fonction de la parure et sur la nature et l'étendue des réseaux d'échanges dans ces sociétés. Les résultats que l'on obtient en étudiant la parure dans ces contextes peuvent également être lus comme des référentiels permettant d'affiner l'analyse d'objets de parure découverts dans des sites pluristratifiés ne permettant pas de discriminer des fréquentations de courte durée.

Dans cette étude nous soumettons les dents perforées découvertes à l'entrée de la Grotte de Praileaitz I, et attribuées au Magdalénien inférieur cantabrique (PEÑALVER, MUJIKA, 2003), à une analyse archéozoologique et technologique dans l'objectif d'affiner l'identification du support (espèce, rang de dents, âge de l'animal), de caractériser les techniques de perforations et de décor, de reconstituer le mode d'attache, la durée d'utilisation et la raison de l'abandon ou de la perte de ces objets. Les résultats de ces analyses prennent toute leur valeur quand ils sont lus à la lumière des informations contextuelles fournies par la fouille de ce site exceptionnel (PEÑALVER, MUJIKA, 2003, PEÑALVER et al., 2017, PEÑALVER et al., 2006, PEÑALVER, SAN JOSE, 2008).

\section{2.- CONTEXTE ARCHÉOLOGIQUE}

Localisée dans une colline calcaire le long de l'avant dernier méandre du Deba, la grotte de Praileaitz I (Deba, Gipuzkoa) a livré trois incisives de bouquetin perforées dans le même niveau que les pendeloques en pierres (D'ERRICO et al., 2017, PEÑALVER et al., 2017) et les blocs d'ocre (D'ERRICO et al., 2017, PEÑALVER et al., 2017). Ce niveau est attribué à un Magdalénien ancien daté par quatre dates ${ }^{14} \mathrm{C}$ et calibrée par IntCal13 (Beta 162880 AMS : $15.190 \pm 50$ BP $(18311-18610$ cal. BP), Beta $162880: 15.300 \pm 50$ BP (18427 - 18720 cal. BP), GrA $20464: 15.460 \pm 100$ BP (18516 - 18917 cal. BP), GrA $24685: 15.530 \pm 100$ BP (18577 - 18979 cal. BP) et

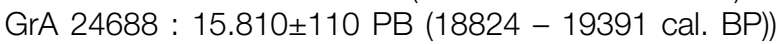
d'environ 15500 BP (PEÑALVER, MUJIKA, 2003, PEÑALVER et al., 2017, PEÑALVER et al., 2006, PEÑALVER, SAN JOSE, 2008), ce qui correspond à un évènement froid appelé « Heinrich 1 » vers la fin du dernier maximum glaciaire (STANFORD et al., 2011). Ces trois incisives de bouquetin ont été découvertes à l'entrée de la grotte dans les carrés C12 et D12 (Fig. 1), tout près de deux grandes pendeloques en pierre décorées trouvées dans le carré B12 (D'ERRICO et al. 2017, PEÑALVER et al., 2017).

\section{3.- MÉTHODES D’ANALYSE}

La latéralisation des incisives de bouquetin transformées en pendentifs et l'estimation de l'âge des animaux a été effectuée en se basant sur la collection d'anatomie comparée du laboratoire PACEA et sur les critères proposés par Couturier (1962) et Ratti et Habermehl (1977). Les dimensions des perforations, la distance entre le centre des deux perforations et la distance entre la perforation centrale et le bord occlusal ont été mesurées avec un pied à coulisse digital. Les quatre faces des incisives ont été digitalisées avec un scanner Epson Perfection à une résolution de 1200 dpi. Les dents ont été observées à la loupe binoculaire pour documenter toute trace d'intervention humaine. Ces traces ont été photographiées à l'aide d'une loupe binoculaire Wild M3C équipée d'une caméra digitale Nikon coolpix 995. Ces photographies ont servi de support pour enregistrer la localisation des traces microscopiques identifiées. Les caractères microscopiques permettant de reconstituer la démarche de l'artisan (traces d'extraction, technique de perforation et production du décor, utilisation du même outil, etc.) et l'utilisation des objets (usures liées au port des objets, traces de pigment etc.) ont également été repérés et documentés photographiquement. L'interprétation de ces traces s'est appuyée sur les critères proposés par d'Errico (1993, 1998), Fritz (1999), Blumenshine et al. (1996), d'Errico et Vanhaeren (1999, 2002).

\section{4.- RÉSULTATS}

Nous avons identifié les trois dents biforées (Fig. 2) comme étant des incisives de bouquetin (Tabl. 1). II s'agit de deux 12/3 gauches (P.A.12C.267.15 et P.A.12C.270.16) et une $11 / 2$ droite (P.A.12D.266.27). Les incisives P.A.12C.270.16 et P.A.12D.266.27 appartiennent certainement au même individu. Cela est suggéré à la fois par le degré d'usure identique de leurs faces occlusales et par deux caractères phénotypiques interprétés comme individuels : des lignes de croissances semblables et très marquées sur la face mésiale et la présence d'une bande plus sombre traversant horizontalement la couronne à la même hauteur sur les deux dents. L'incisive P.A.12C.267.15 ne possède pas ces caractères et la facette d'usure qu'elle présente sur sa face mésiale n'a pas de correspondant sur la face distale de l'incisive P.A.12C.270.16, incisive qu'elle aurait pourtant côtoyée si elles provenaient du même individu.

L'âge des deux bouquetins dont proviennent ces dents peut être estimé en considérant l'état d'usure de la surface occlusale et le dégrée de fermeture de la cavité pulpaire. Chez le bouquetin, les I2/I3 remplacent les incisives déciduales entre 28 et 38 mois (COUTURIER, 1962, RATTI, HABERMEHL, 1977). Une facette d'usure 
Fig. 2. Haut : quatre faces des trois incisives de bouquetin biforées découvertes à Praileaitz I. Bas : relevé des traces anthropiques identifiées sur ces dents (bleu : traits gravés ; vert : encoches, gris : traces de découpe ; rouge : traces d'usure). Échelle $=1$ $\mathrm{cm}$. / Top: four aspects of each of the three biforated ibex incisors from Praileaitz I. Bottom: drawing indicating the location of the modifications identified on these teeth (blue: engraved lines, green: notches, grey: cut marks, red: use-wear). Scale $=1 \mathrm{~cm}$.
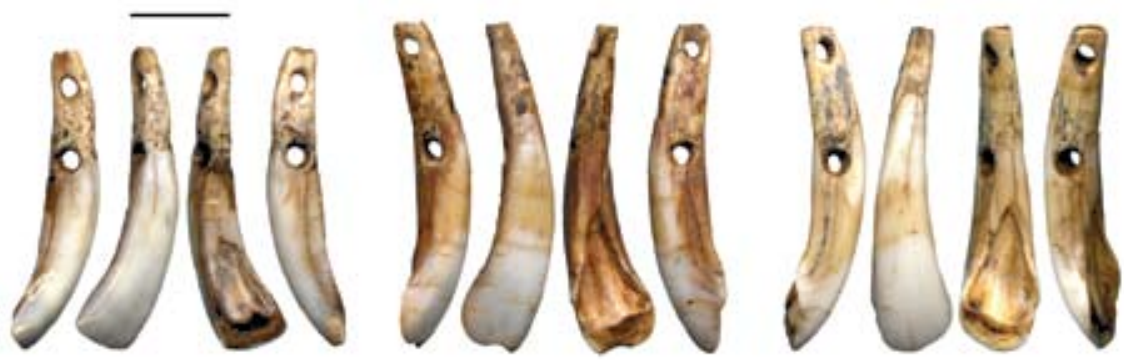

P.A.12C.267.15
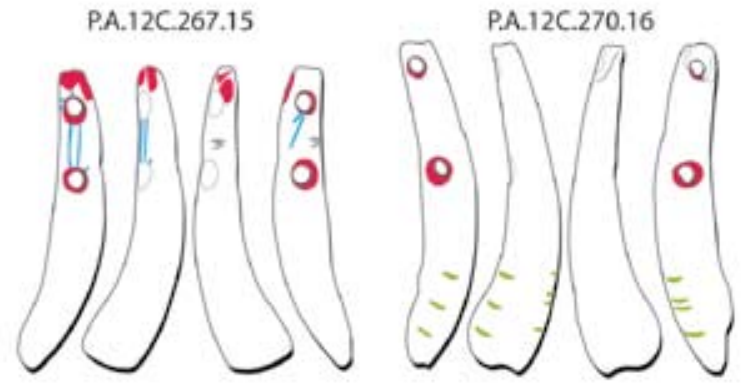

P.A.12D.266.27

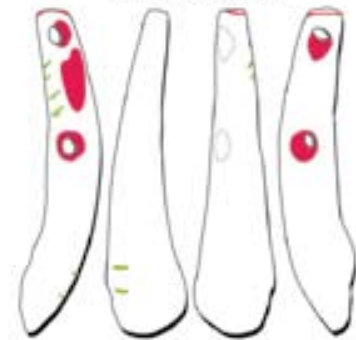

\begin{tabular}{|c|c|c|c|c|c|c|c|c|c|c|}
\hline \multirow{3}{*}{ N. } & \multirow{3}{*}{$\begin{array}{c}\text { Rang } \\
\text { dentaire }\end{array}$} & \multicolumn{4}{|c|}{ Dimension des perforations } & \multicolumn{2}{|c|}{ Distance } & \multicolumn{2}{|c|}{ Décor } & \multirow{3}{*}{$\begin{array}{c}\text { Usure } \\
\text { localisation }\end{array}$} \\
\hline & & \multicolumn{2}{|c|}{ collet } & \multicolumn{2}{|c|}{ apex } & \multirow{2}{*}{$\begin{array}{l}\text { P au collet- } \\
\text { P à l'apex } \\
\text { (mm) }\end{array}$} & \multirow{2}{*}{$\begin{array}{l}\text { P au collet- } \\
\text { bord occlusal } \\
(\mathrm{mm})\end{array}$} & \multirow[b]{2}{*}{ traits } & \multirow[b]{2}{*}{ entailles } & \\
\hline & & $\begin{array}{l}\text { Dmax } \\
(\mathrm{mm})\end{array}$ & $\begin{array}{l}\text { Dmin } \\
(\mathrm{mm})\end{array}$ & $\begin{array}{l}\text { Dmax } \\
(\mathrm{mm})\end{array}$ & $\begin{array}{l}\text { Dmin } \\
(\mathrm{mm})\end{array}$ & & & & & \\
\hline P.A.12C.267.15 & $12 / 3$ gauche & 1,88 & 1,67 & 1,96 & 1,63 & 7,75 & 19,17 & ? & non & $P, A$ \\
\hline P.A.12C.270.16 & $12 / 3$ gauche & 1,88 & 1,67 & 1,71 & 1,42 & 10,88 & 19,16 & oui & oui & $\mathbf{P}$ \\
\hline P.A.12D.266.27 & 11/2 droite & 1,83 & 1,79 & 1,75 & 1,25 & 11,63 & 18,67 & oui & oui & $\mathrm{P}, \mathrm{FM}, \mathrm{A}$ \\
\hline
\end{tabular}

P: perforation; A: apex, FM: face mésiale; Dmax : Diamètre maximal, Dmin : Diamètre minimal

Tabl. 1. Informations concernant les incisives de bouquetin perforées de Praileaitz I. / Analytical data on the perforated ibex incisors from Praileaitz I.

concave qui laisse apparaître, comme dans notre cas, la dentine jaunâtre, se développe un an après l'éruption de l'incisive permanente. Le foramen apical s'obture, quant à lui, avant l'âge de 6 ans sur les 11-3. L'âge des deux bouquetins dont proviennent ces trois incisives doit être compris entre 3,5 et 6 ans. II s'agit donc de jeunes adultes.

L'étude microscopique de la dent P.A.12C.267.15 (Fig. 3) révèle sur la face mésiale deux incisions parallèles, produites par la même pointe, qui relient les deux perforations et qui semblent avoir été gravées avant celles-ci (Figs. 2 et $3 a-c)$. Une strie semblable aux précédentes traverse obliquement la face distale (Figs. 2 et $3 d-e$ ). Deux incisions, courtes, produites par un tranchant, sont présentes sur le bord disto-linguale (Figs. 2 et 3e). De rares incisions rayonnent du bord de la perforation proche de l'apex (Fig. 3a,b). Ces dernières sont probablement dues à une préparation de la surface pour caler la pointe, préalablement à la perforation de la racine, obtenue par rotation bipolaire d'une pointe lithique. Les traits qui relient les deux perforations semblent être le résultat d'une gravure intentionnelle plutôt que d'un procédé technique destiné à réduire l'épaisseur de la racine. Les stries restantes sont difficiles à interpréter. Elles ressemblent à des stries de découpe et pourraient avoir été laissées au cours de l'extraction de la dent. Les stries de rotation sur les bords des perforations de cette dent sont presque entièrement effacées par l'usure due au lien. L'usure crée également une zone polie entre la perforation apicale et l'extrémité de l'apex (Fig. 3b).

La dent P.A.12C.270.16 a été légèrement raclée avec une pointe lithique, au niveau du collet, sur ses faces mésiale et distale (Fig. 4c, f). La racine a été ensuite perforée par rotation bipolaire. Les perforations apparaissent, sur cette dent, moins usées que sur la dent P.A.12C.267.15. De plus, celle proche de l'apex (Fig. 4a) présente une usure plus intense que celle située près du collet (Fig. 4c). La couronne a été décorée avec trois encoches sur son bord mesio-vestibulaire et quatre sur son bord disto-vestibulaire (Figs. 2 et $5 \mathrm{a}$ ). Ces encoches ont été produites par le mouvement de va et vient d'un 

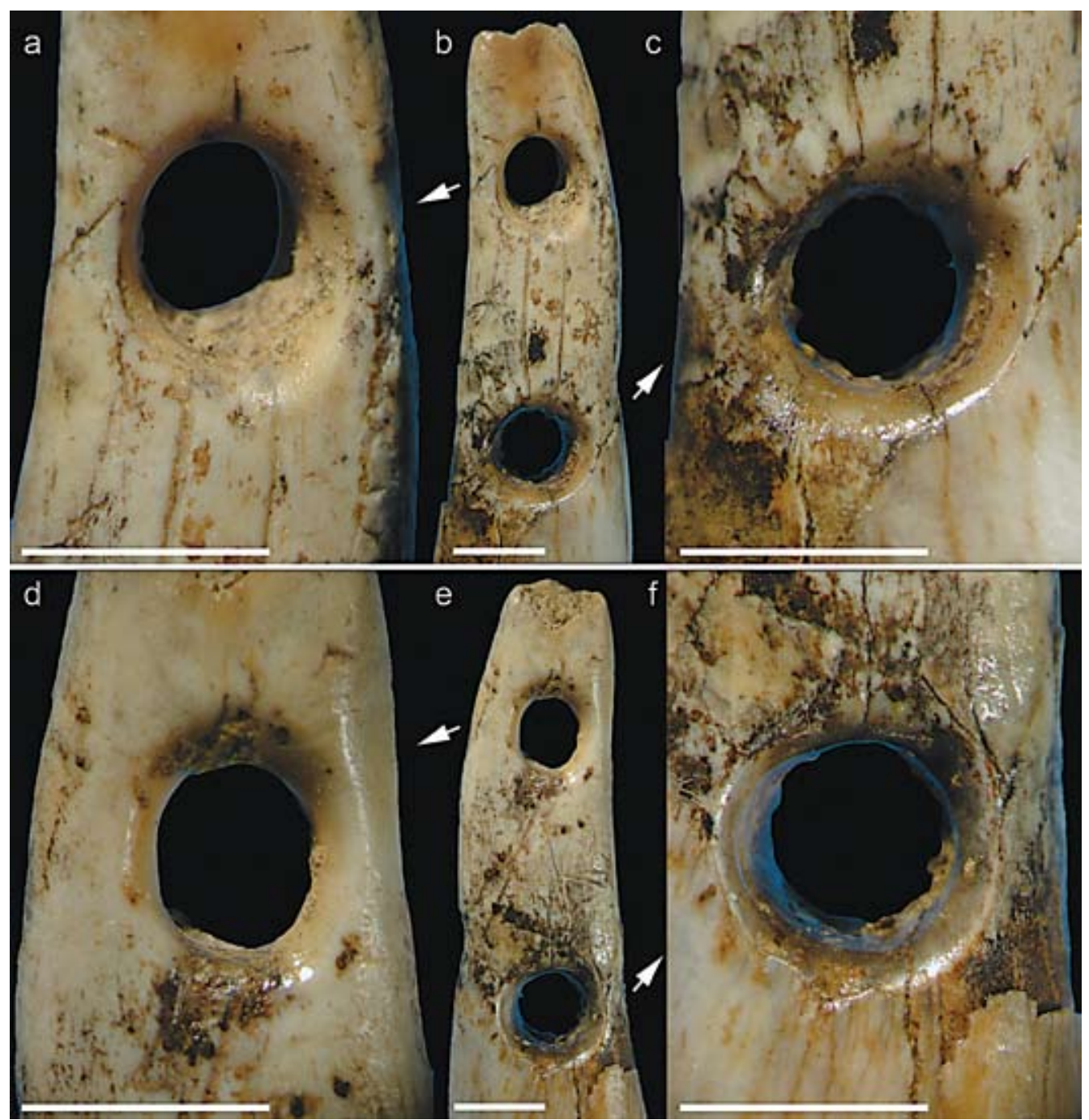

Fig. 3. Macrophotos des faces distale (a-c) et mésiale (d-f) de l'incisive de bouquetin P.A.12C.267.15 de Praileaitz I. Échelles $=250 \mu \mathrm{m}$. / Macrophotos of the distal (a-c) and mesial aspects (d-f) of the ibex incisor P.A.12C.267.15 from Praileaitz I. Scales $=250 \mu \mathrm{m}$.

même tranchant, non retouché. Des résidus de pigment rouge ont été détectés sur la racine et la couronne de cette dent (Fig. 4). Leur présence à l'intérieur des encoches (Fig. 5a) suggère que celles-ci devaient à l'origine être mises en exergue par le pigment.

La racine de la dent P.A.12D.266.27 présente sur son bord mésio-lingual une série de stries de découpe obliques, à mettre probablement en relation avec l'extraction de la dent (Figs. 2 et 6b). Comme dans le cas précédent les perforations ont été réalisées par rotation et celle proche de l'apex (Fig. 6a, d) apparaît plus usée que celle réalisée sur le collet (Fig. 6c, f). La face mésiale de la racine est affectée par une usure qui aplanit sa surface (Fig. 2). Le bord mesio-vestibulaire de la couronne est décoré avec deux encoches réalisées par le même tranchant lithique non retouché (Fig. 5b). Des résidus microscopiques de pigment rouge sont présents sur la face mésiale de la racine et autour de la perforation centrale.
Les variables métriques révèlent une remarquable homogénéité dans la taille des six perforations présentes sur ces incisives et dans la distance entre les perforations situées sur le collet et le bord occlusal (Tabl. 1). II en est de même pour la distance entre les perforations sur les dents P.A.12C.270.16 et P.A.12D.266.27, celle sur la dent P.A.12C.267.15 étant, par contre, nettement inférieure (Tabl. 1).

\section{5.- DISCUSSION ET CONCLUSION}

Les dents P.A.12C.270.16 et P.A.12D.266.27 se distinguent de la dent P.A.12C.267.15 pour avoir été prélevées sur le même individu, posséder des perforations équidistantes, avoir des couronnes décorées avec des encoches, présenter des perforations avec le même patron d'usure et conserver des résidus de pigment rouge. 

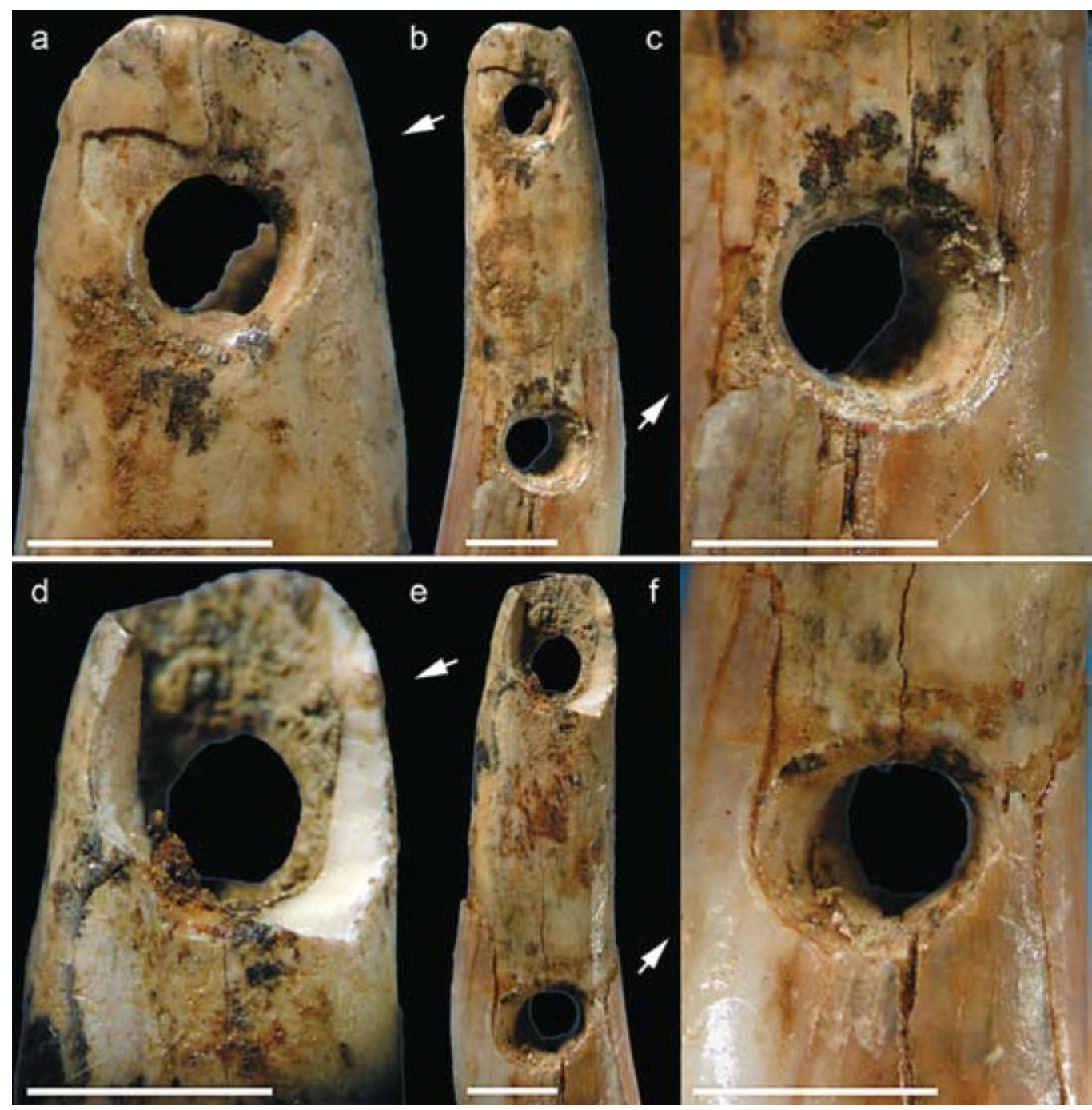

Fig. 4. Macrophotos des faces distale (a-c) et mésiale (d-f) de l'incisive de bouquetin P.A.12C.270.16 de Praileaitz I. Échelles $=250 \mu \mathrm{m}$. / Macrophotos of the distal $(\mathrm{a}-\mathrm{c})$ and mesial aspect $(\mathrm{d}-\mathrm{f})$ of the ibex incisor P.A.12C.270.16 from Praileaitz I. Scales $=250 \mu \mathrm{m}$.
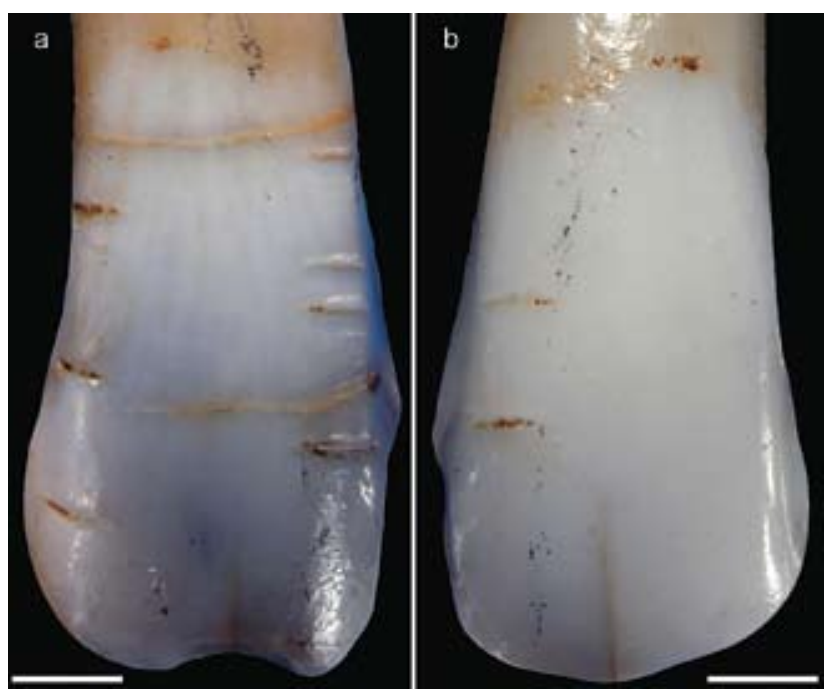

II est très probable, à la lumière de ces observations, que ces deux pendentifs on été fabriqués et décorés par la même personne, qu'ils étaient intégrés à l'origine dans la même parure et qu'ils ont été abandonnés ou perdus au même moment. Même s'il est difficile de le démontrer, la dent P.A.12C.267.15 pourrait également faire partie de la même parure en considérant sa proximité spatiale avec les deux autres incisives et

Fig. 5. Macrophotos des couronnes décorées des incisives de bouquetin P.A.12C.270.16 (a) et P.A.12D.266.27 (b) de Praileaitz I. Échelles $=250 \mu \mathrm{m}$. $/$ Macrophotos of the decorated tooth crowns of ibex incisors P.A.12C.270.16 (a) and P.A.12D.266.27 (b) from Praileaitz I. Scales $=250 \mu \mathrm{m}$ 

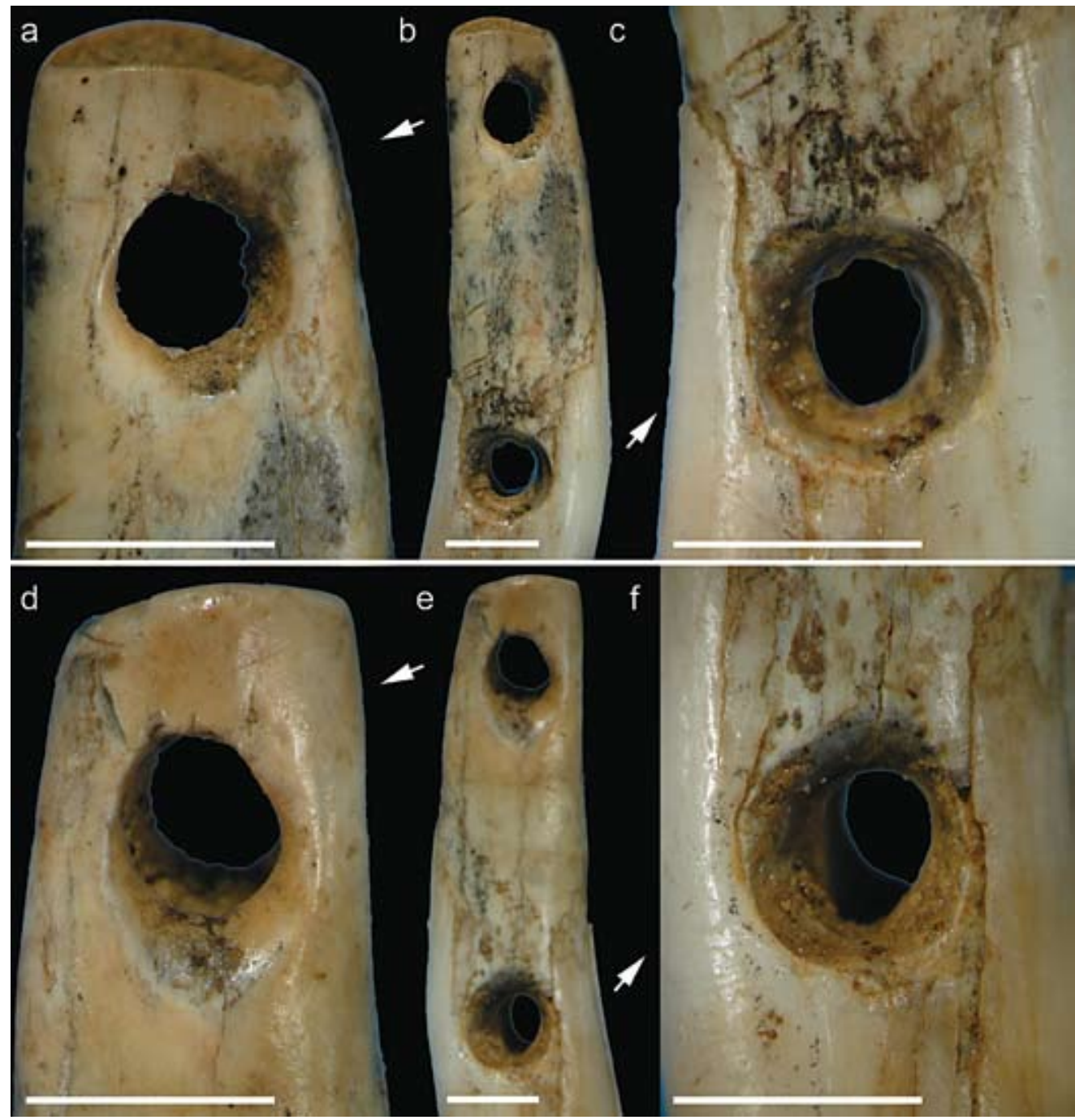

Fig. 6. Macrophotos des faces distale (a-c) et mésiale ( $d-f)$ de l'incisive de bouquetin P.A.12D.266.27 de Praileaitz I. Échelles $=250 \mu \mathrm{m}$. / Macrophotos of the distal $(\mathrm{a}-\mathrm{c})$ and mesial aspect $(\mathrm{d}-\mathrm{f})$ of the ibex incisor P.A.12D.266.27 from Praileaitz I. Scales $=250 \mu \mathrm{m}$

la forte ressemblance dans la technique et la taille des perforations. Les trois incisives perforées de bouquetin découvertes dans les couches gravettiennes d'Aitzbitarte représentent, à notre connaissance, le seul cas connu de dents de cette espèce pour lesquelles il a été montré qu'elles provenaient du même bouquetin et avaient probablement été perforées par le même artisan (D'ERRICO, VANHAEREN, 1999). Le fait que la variabilité dimensionnelle des perforations sur les dents de Praileaitz soit inférieure à celle observée à Aitzbitarte penche pour une attribution de la dent P.A.12C.267.15 au même collier. II est intéressant aussi de remarquer à ce propos que la perforation centrale est située, sur les trois dents de Praileaitz, exactement à la même distance du bord occlusal (Tabl. 1), choix permettant d'aligner les bords occlusaux dans un dispositif de parure dans lequel les incisives sont juxtaposées. Ce fait penche également pour une intégration de la dent P.A.12C.267.15 au sein du même dispositif de parure. Les quelques différences observées dans le degré d'usure des perforations ne contredisent pas l'hypothèse d'une provenance du même collier. Elles peuvent dépendre de la localisation des objets au sein du dispositif de parure et de l'intensité des frottements qui en découlent. En somme, nos résultats montrent que ces incisives faisaient vraisemblablement partie d'un même dispositif de parure (collier, bracelet, bandeau etc.) arrivé sur le site en tant qu'objet fini et déjà usé. Le fait que toutes les perforations soient entières suggère que la cassure du fil d'attache est la raison la plus probable pour la perte de ces incisives. II est logique de penser que l'objet d'origine devait être composé d'un nombre plus important d'incisives et que lors de sa fracture certains ont été récupérés en vue de leur réutilisation et d'autres irrémédiablement perdus. Ces résultats s'accordent avec l'hypothèse d'une occupation ponctuelle de la cavité au cours du Magdalénien inférieur cantabrique. 


\section{6.- REMERCIEMENTS}

Nous remercions Xabier Peñalver, Solange Rigaud et Alain Queffelec pour la relecture critique de ce manuscrit et Jean-Baptiste Mallye, Myriam Boudadi-Malligne et Delphine Kuntz pour les discussions sur les restes de bouquetin. Cette recherche a été financée par le CNRS français et le programme de l'European Science Foundation the Origin of Man, Language and Languages.

\section{7.- BIBLIOGRAPHIE}

ÁLVAREZ-FERNANDEZ, E.

2006 Los objetos de adorno-colgantes del Paleolitico superior y del Mesolitico en la Cornisa Cantábrica y en el Valle del Ebro: una vision europea. Universidad de Salamanca. Salamanca.

\section{BLUMENSCHINE, J., MAREAN, C., CAPALDO, S.D.}

1996 Blind tests of interanalyst correspondence and accuracy in the identification of cut marks, percussion marks, and carnivore tooth marks on bone surfaces. Journal of Archaeological Science 2, 493-507.

BONNARDIN, S.

2009 La parure funéraire au Néolithique ancien dans les Bassins parisiens et rhénans - Rubané, Hinkelstein et VilleneuveSaint-Germain. Société Préhistorique Française, Paris. (Mémoires de la Société préhistorique française, 49).

COUTURIER, M.

1962 Le bouquetin des Alpes. Allier. Grenoble.

D'ERRICO, F.

1993 La vie sociale de l'art mobilier paléolithique. Manipulation, transport, suspension des objets en os, bois de cervidés, ivoire. Oxford Journal of Archaeology 12(2), 145-174.

1998 Palaeolithic origins of artificial memory systems: an evolutionary perspective, en RENFREW, C., SCARRE, C. (Eds.). Cognition and Material Culture: the Archaeology of Symbolic Storage. 19-50. McDonald Institute. Cambridge. (McDonald Institute Monographs).

D'ERRICO, F., VANHAEREN, M.

1999 Les méthodes d'analyse de l'art mobilier paléolithique. Quelques exemples issus de la région cantabrique. Anthropologie et préhistoire 110, 31-45.

2002 Criteria for identifying red deer age and sex from their canines. Application to Upper Palaeolithic and Mesolithic ornaments. Journal of Archaeological Science 29, 211-232.

D'ERRICO, F., VANHAEREN, M., QUEFFELEC, A.

2017 Les galets perforés de Praileaitz I (Deba, Gipuzkoa), en PEÑALVER, X., SAN JOSE, S., MUJIIKA-ALUSTIZA, J.A. La cueva de Praileaitz I (Deba, Gipuzkoa, Euskal Herria). Intervención arqueológica 2000 - 2009. Munibe Monographs. Anthropology and Archaeology Series 1, 453484.
FRITZ, C.

1999 La gravure dans l'art mobilier magdalénien. Du geste à la représentation. DAF. Paris. (Documents d'archéologie française 75).

HENSHILWOOD, C., D'ERRICO, F.

2005 Being Modem in the Middle Stone Age: individuals and innovation, en GAMBLE, C., POOR, M. (Eds.). Individuals in the Paleolithic. 244-264. Routledge University Press. London.

PEÑALVER IRIBARREN, X., MUJIIKA ALUSTIZA, J.A.

2003 Suelo de ocupación magdaleniense en la cueva de Praile Aitz I (Deba, Gipuzkoa): evidencias de arte mobiliar. Veleia 20, 157-81.

PEÑALVER IRIBARREN, X., SAN JOSE SANTAMARTA, S.

2008 La grotte de Praileaitz I (Deba, Gipuzkoa, Euskal Herria Pays Basque et la défense du patrimoine dans le Pays Basque). Préhistoire du Sud-Ouest 16, 143-50.

PEÑALVER, X., SAN JOSE, S., MUJIKA-ALUSTIZA, J.A.

2017 La cueva de Praileaitz I (Deba, Gipuzkoa, Euskal Herria). Intervención arqueológica 2000 - 2009. Munibe Monographs. Anthropology and Archaeology Series, 1.

PEÑALVER, X., SAN JOSE, S., MUJIKA, S.A., XABI OTERO, A.

2006 Praileaitz I Haitzuloko (Deba) Madeleine Aldiko Zintzilikarioak. Gipuzkoako Foru Aldundia. Deba.

RATTI, P. VON, HABERMEHL, K.-H.

1977 Untersuchungen zur Altersschätzung und Altersbestimmung beim Alpensteinbock (Capra ibex ibex) im Kanton Graubünden. Zeitschrift zur Jagdwissenschaft, 188-213.

RIGAUD, S.

2011 La parure : traceur de la géographie culturelle et des dynamiques de peuplement au passage Mésolithique-Néolithique en Europe. Université Bordeaux 1. Talence.

STANFORD, J.D., ROHLING, E.J., BACON, S., ROBERTS, A.P., GROUSSET, F.E., BOLSHAW, M.

2011 A new concept for the paleoceanographic evolution of Heinrich event 1 in the North Atlantic. Quaternary Science Reviews 30(9-10), 1047-1066.

VANHAEREN, M.

2002 Les fonctions de la parure au Paléolithique supérieur : de l'individu à l'unité culturelle. Université de Bordeaux 1. Talence.

VANHAEREN, M., D'ERRICO, F.

2003 The Body Ornaments Associated with the Burial, en ZUHÃO, J., TRINKAUS, E. (Eds.), Portrait of the Artist as a Child. The Gravettian human skeleton from the Abrigo do Lagar Velho and its archaeological context. 154-186. Instituto Portugues de Archeologia. Lisboa. (Trabalhos de Arqueologia 22). 